\title{
ON HARMONIC CONTENT OF THE WAKE BEHIND A CIRCULAR
} CYLINDER

\author{
Uruba V.*, Procházka P.**
}

\begin{abstract}
The wake behind cylinder of circular cross-section is subjected to detailed analysis from the point of view harmonic content. The basic, fundamental frequency could be linked to well-known Bénard-von Kármán vortex street, it is called Strouhal frequency very often. However higher harmonics of $2^{\text {nd }}$ and $3^{\text {rd }}$ order have been detected, as well as important inharmonic partials. The topologies of the velocity flow-field corresponding to the harmonic is to be shown.
\end{abstract}

Keywords: Circular cylinder, Wake, Strouhal number, Higher harmonics, Oscillation Pattern Decom-
position.

\section{Introduction}

The wake behind a circular cylinder belongs to so called canonical cases, well investigated by all means available. Many experimental studies and mathematical simulations could be find in literature (see e.g. Roshko, 1955, Williamson 1996).

The wake dynamical behavior is presented to be governed by the fundamental frequency, which characterize periodical separation of vortical structures, so called von Kármán-Bénard vortex street. The dimensionless frequency is called Strouhal number $S t$ and it is defined as follows: $S t=f \cdot d / U_{i}$, where $f$ is the fundamental frequency, $d$ is the cylinder diameter and $U_{i}$ is incoming velocity. Typical value of the Strouhal number is about $S t=0.2$ in the broad range of Reynolds numbers, higher than 200 (see e.g. Roshko, 1955).

The dynamics of the cylinder wake is considered to be turbulent (see e.g. Uruba, Procházka, 2019) with a single predominant frequency peak on the fundamental frequency. However, the flowing fluid represents itself strongly non-linear dynamical system, this fact is demonstrated by Navier-Stokes equations. From the theory of non-linear dynamical systems it is known that the higher harmonics should appear in the system dynamics as a rule. However, presence of higher harmonics in the wake have not been studied yet.

The presented study brings experimental evidence of higher harmonics presence in the wake flow-field dynamics.

\section{Experimental Setup and Data Analysis Methods}

The experimental setup and procedures are to be described. The phenomenon is considered to be statistically $2 \mathrm{D}$, the position of observation along the cylinder axis is insignificant. This assumption follows from the fact that the boundary conditions are 2D as well. However, it has been proved that the instantaneous velocity field is fully 3D (see Uruba, 2016), this is the basic attribute of any turbulent flow. The wake three-dimensionality was studied e.g. in Uruba, Procházka, Skála (2020).

\footnotetext{
* Prof. Ing. Václav Uruba, CSc.: Institute of Thermomechanics of the Czech Academy of Sciences, Dolejškova 5, Praha 8, CR, uruba@it.cas.cz, University of West Bohemia, Faculty of Mechanical Engineering, Department of Power System Engineering, Universitní 8, Plzeň, CR, uruba@kke.zcu.cz

** Ing. Pavel Procházka, PhD.: Institute of Thermomechanics of the Czech Academy of Sciences, Dolejškova 5, Praha 8, CR, prochap@it.cas.cz
} 


\subsection{Experimental Setup}

The cylinder of diameter $15 \mathrm{~mm}$ is placed perpendicularly to the flow in the test-section inlet of the blowndown facility. The cross-section of the closed test-section is $250 \times 250 \mathrm{~mm}^{2}$ in the position of the cylinder. The velocity on the test-section inlet was $U_{i}=5 \mathrm{~m} / \mathrm{s}$ corresponding to the Reynolds number about 4815 . The Cartesian coordinate system was introduced, origin is located on the cylinder axis, see Fig. 1.

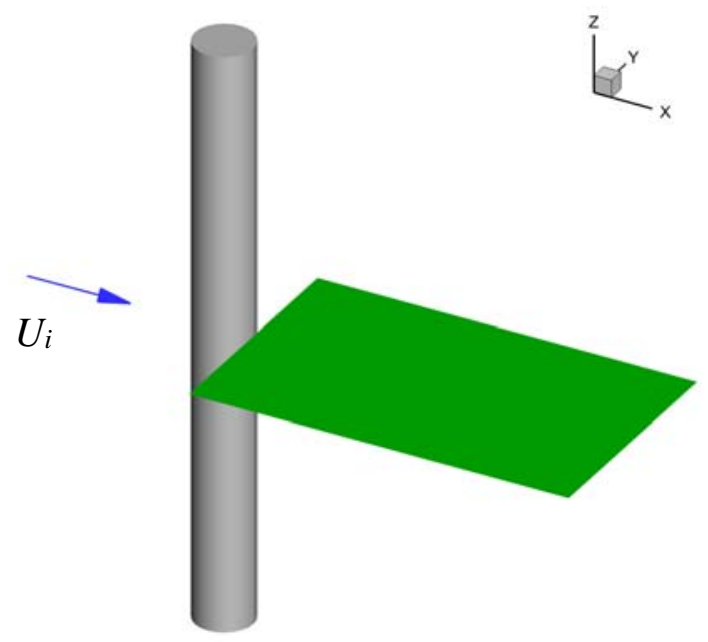

Fig. 1: Model and plane of measurement.

The velocity vector field was measured using Particle Image Velocimetry (PIV) method. The measurement apparatus consists of laser and CMOS camera by Dantec company. The laser is New Wave Pegasus, Nd:YLF double head with wavelength of $527 \mathrm{~nm}$, maximal frequency $10 \mathrm{kHz}$, shot energy is $10 \mathrm{~mJ}$ (for $1 \mathrm{kHz}$ ) and corresponding power is $10 \mathrm{~W}$ per one head. The camera Phantom V611 with resolution of $1280 \times 800$ pixels is able to acquire double snaps with frequency up to $3000 \mathrm{~Hz}$ (full resolution) and it uses internal memory $8 \mathrm{~GB}$. The data were acquired and post-processed in Dynamic Studio software.

The PIV measurement was performed in the plane perpendicular to the cylinder axis and parallel to the flow direction $(x y)$, shown in green in Fig. 1. The evaluated velocity fields consisting of $159 \mathrm{x} 99$ vectors were acquired in two different regimes. The dynamical analysis was performed on data acquired by frequency $2 \mathrm{kHz}$, one record contained 4000 double-snapshots representing $2 \mathrm{~s}$ in real time.

\subsection{Data Analysis Methods}

The Oscillation Pattern Decomposition method (hereinafter OPD) is used to study the dynamical properties of the flow-field. The OPD method yields series of OPD modes. Each OPD mode is characterized by its topology in complex form (consisting of real and imaginary parts), frequency and attenuation of the pseudoperiodic (oscillating) behavior. Attenuation or amplitude decay is described by so called e-folding time $\tau_{\mathrm{e}}$ representing the mean time period of the mode amplitude decay by factor "e". The other decay characteristic is dimensionless "periodicity" $p$ which expresses the e-folding time in multiples of periods of the OPD mode defining by its frequency. The details on OPD method could be found in Uruba $(2012,2015)$.

\section{Results}

The dimensionless coordinates $(x y z)$ are used, the origin of the coordinate system is on the cylinder axis, dimensions are expressed in multiples of the cylinder diameter $d$. Velocity vectors and vorticity are also dimensionless, appropriated arbitrary scaling is used.

\subsection{OPD Analysis}

In Tab. 1 the 3 most important OPD modes are introduced. 12 modes have been evaluated, however higher order modes are unimportant, with small periodicity. The frequency for each mode $f$, e-folding time $\tau_{\mathrm{e}}$ and periodicity $p$ are shown for the OPD modes. In addition, the Strouhal number $S t$ is given and the harmonic order $n$, the fundamental frequency is assigned to be the frequency of the OPD mode 1 . The modes are ordered according to the periodicity value, the higher periodicity, the more important the mode is. 
The first 3 POD modes, which are the dominant, represent the first, third and second harmonics respectively. The higher OPD modes are weak, rapidly decaying, the frequencies belong to inharmonic partials. They represent rather turbulent features of the wake.

Tab. 1: The 3 harmonic OPD modes.

\begin{tabular}{|c|c|c|c|c|c|}
\hline Mode No. & $f[\mathrm{~Hz}]$ & $\tau_{\mathrm{e}}[\mathrm{s}]$ & $p[1]$ & St $[1]$ & $n[1]$ \\
\hline 1 & 70.7 & 0.4229 & 29.886 & 0.212 & 1.000 \\
\hline 2 & 210.3 & 0.0426 & 8.965 & 0.631 & 2.975 \\
\hline 3 & 135.6 & 0.0217 & 2.938 & 0.407 & 1.919 \\
\hline
\end{tabular}

\subsection{Fundamental Frequency}

The complex topology of the OPD modes is to be presented, in Fig. 2 the OPD mode 1 is shown. The real part of the OPD mode represents situation for the phase angle 0 , the imaginary part corresponds to phase $\pi / 2$, then negative real for $\pi$, negative imaginary for $3 \pi / 2$ and again positive real part for phase $2 \pi(0)$.
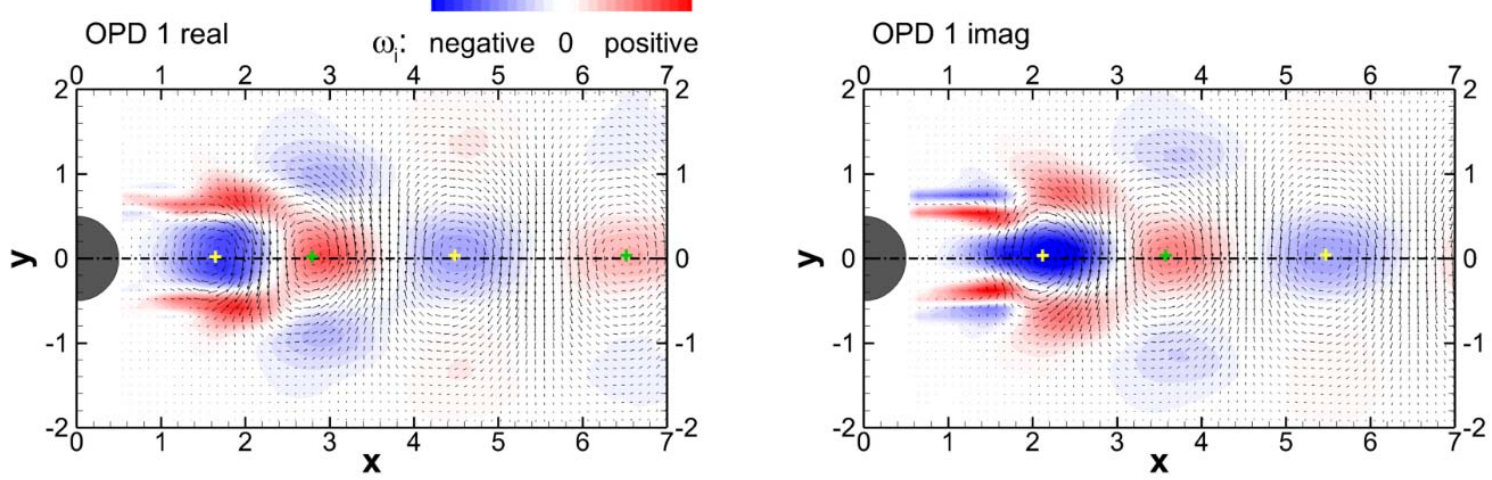

Fig. 2: OPD mode 1 topology on fundamental frequency, real (left) and imaginary (right) parts.

The arrows represent velocity vectors, color corresponds to vorticity component $\omega_{z}$ perpendicular to the (xy) plane, red positive, blue negative, white close to zero. The vortices are detected in the flow patterns, centers are marked by crosses, positive in green and negative in yellow.

The OPD mode 1 representing the fundamental frequency periodical behavior of the system, consists of a train of vortices of alternating orientation with centers located just on the $x$ axis. Some minor secondary structures could be detected as well.

\subsection{Higher Harmonics}

The OPD modes 2 and 3 represents $3^{\text {rd }}$ and $2^{\text {nd }}$ harmonic contents respectively, they are shown in Figs. 3 and 4.
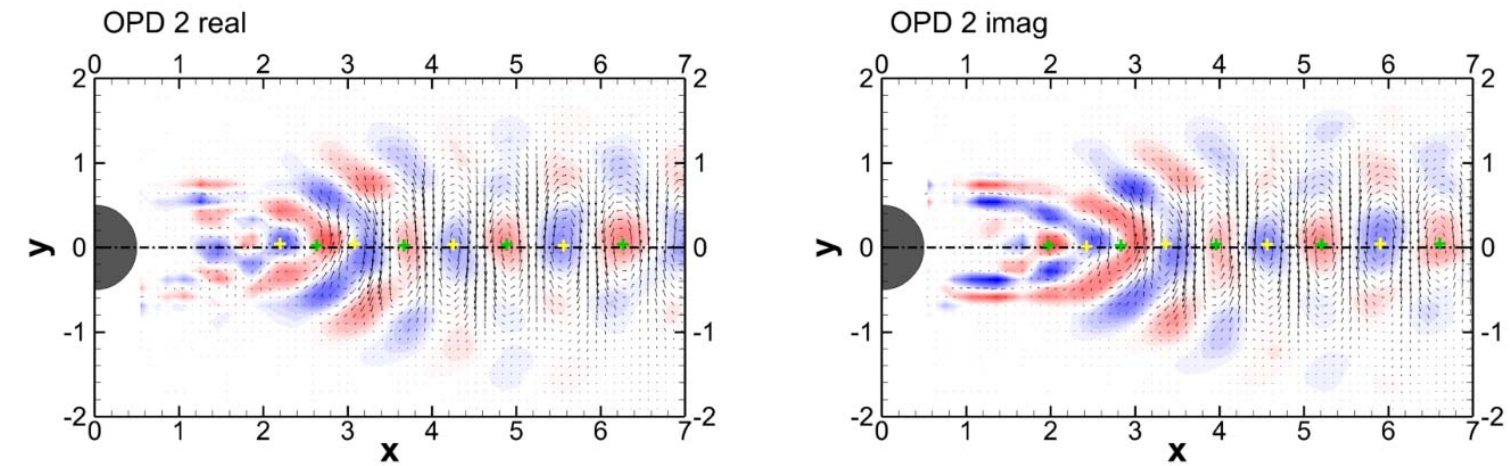

Fig. 3: OPD mode 2 topology on the $3^{\text {rd }}$ harmonics, real (left) and imaginary (right) parts. 

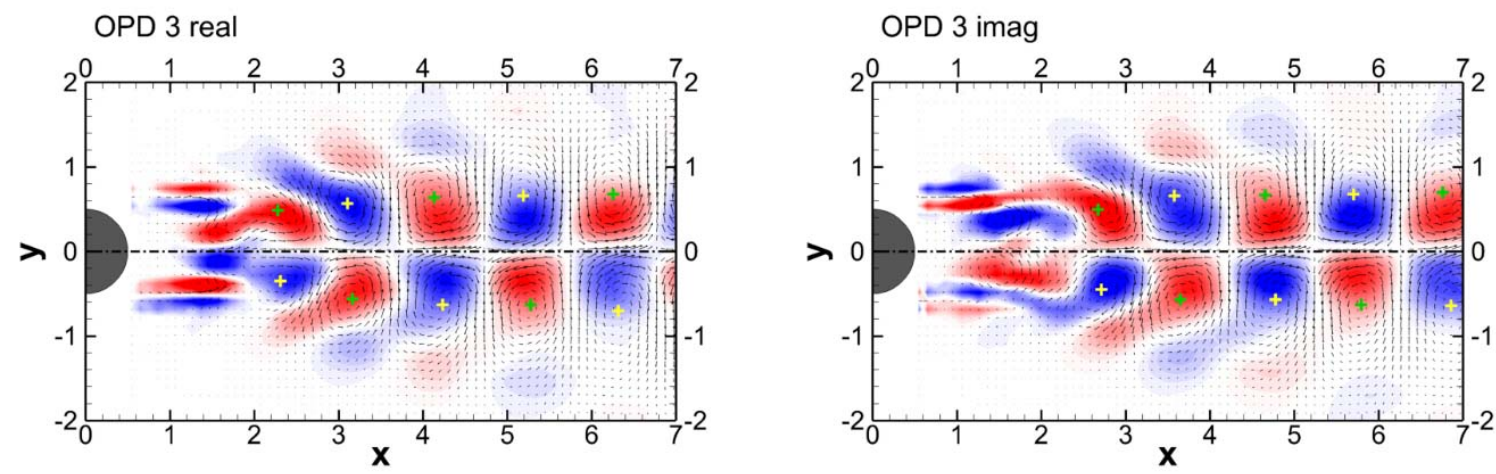

Fig. 4: OPD mode 3 topology on the $2^{\text {nd }}$ harmonics, real (left) and imaginary (right) parts.

The OPD mode 2 topology on the $3^{\text {rd }}$ harmonics is represented by vortices with alternate orientation again aligned with the $x$ axis, similar to the fundamental frequency OPD mode 1, however much smaller spacing. The OPD mode 3 shows the topology for the $2^{\text {nd }}$ harmonics, which is different. It consists of two rows of vortices forming a checkered pattern. The vortices are very strong and good pronounced with alternative orientation.

The convection velocity of the vortices could be evaluated from the vortex centers distribution and period of the corresponding periodical process, it is between $50 \%$ and $90 \%$ of the incoming velocity $U_{\mathrm{i}}$, lower just behind the cylinder.

\section{Conclusions}

The harmonic content of the wake behind a circular cylinder at Reynolds number of about 5 thousands has been studied using OPD method.

Besides the fundamental Strouhal frequency, the $2^{\text {nd }}$ and $3^{\text {rd }}$ harmonics have been detected. The flow patterns reveal the typical vortex trains in the wake carried by the flow. The fundamental and the $3^{\text {rd }}$ harmonics are represented by vortices of alternating orientation with centers located on the flow-axis, while the $2^{\text {nd }}$ harmonics consists of two rows of vortices forming a checkered pattern.

The convection velocity of all vortical structures is approximately the same and ranges from $50 \%$ to $90 \%$ of the incoming flow velocity.

The higher order OPD modes represent turbulent rapidly decaying inharmonic partials.

\section{Acknowledgement}

This work was supported by the Grant Agency of the Czech Republic, projects Nos. 17-01088S, 19-04695S and $19-02288 \mathrm{~J}$.

\section{References}

Roshko, A. (1955) On the development of turbulent wakes from vortex streets. NACA Rep. 1191, pp. 801-825.

Uruba, V. (2012) Decomposition methods in turbulent research, EPJ Web of Conferences, vol. 25, 01095.

Uruba, V. (2015) Near Wake Dynamics around a Vibrating Airfoil by Means of PIV and Oscillation Pattern Decomposition at Reynolds Number of 65 000, Journal of Fluids and Structures, 55, pp. 372-383.

Uruba, V. (2016) On 3D instability of wake behind a cylinder, AIP Conference Proceedings, Vol 1745, Art. no. 020062

Uruba, V., Procházka, P. (2019) The Reynolds number effect on dynamics of the wake behind a circular cylinder. AIP Conference Proceedings, vol. 2189, Article no. 020023.

Uruba, V., Procházka, P., Skála, V. (2020) On the 3D Dynamics of the Wake Behind a Circular Cylinder, In Proceedings Topical Problems of Fluid Mechanics 2020, Prague, Ed. D. Šimurda and T. Bodnár, pp. 240-248.

Williamson, C. H. K. (1996) Vortex Dynamics in the Cylinder Wake, Annu. Rev. Fluid. Mech. vol. 28, pp. 477-539. 